\title{
应力波作用下岩石类孔隙介质变形破坏与 能量耗散机制的数值模拟研究
}

鞠杨 ${ }^{(1)}$ ，王会杰 ${ }^{(1)}$, 杨永明 ${ }^{(1)}$, 胡沁昂 ${ }^{(2)}$, 彭瑞东 ${ }^{(1)}$

(1) 中国矿业大学煤炭资源与安全开采国家重点实验室, 北京市岩石混凝土破坏力学重点实验室, 北京 100083;

(2) School of Environment and Civil Engineering, College of Engineering, Oklahoma State University, 207 Engineering South, Stillwater, OK 74078, USA

*E-mail: juy@cumtb.edu.cn

收稿日期: 2009-09-06; 接受日期: 2009-11-19

国家重点基础研究发展计划(“973”计划)(批准号: 2010CB226804, 2002CB412705)、国家自然科学基金(批准号: 50974125)、北京市重点实验室项目资助

摘要通过 CT 扫描、X 射线衍射和物理实验等方法获取了天然砂岩的孔隙结构参数、矿物 组成和物理力学性质, 研制了具有与天然砂岩相同的孔隙结构特征和基体性质、但孔隙率不同 的岩石类孔隙介质的物理模型. 利用孔隙介质物理模型的 CT 扫描图像和 MIMICS 构建了具有 不同孔隙率的孔隙结构三维有限元模型. 通过设定应力波动理论假设的条件模拟了孔隙介质 SHPB 冲击破坏过程, 分析了波动应力作用下岩石类孔隙介质的动力学响应、应力传递模式和 变形破坏机制. 研究表明: 利用孔隙介质三维有限元模型可以直观定量地分析应力波传播过程 中岩石类介质内部孔隙和基体的应力、应变状态及变形破坏机制。一定压强和波速的应力波传 播过程中, 孔隙率低于 $15 \%$ 的岩石介质内部的孔隙未发生明显变形, 变形主要体现为孔隙周边 基体的微塑性(剪切变形)和开裂(横向拉应变), 以及孔隙周边开裂区域的相互连通. 剪应力使基 体单元产生微塑性, 拉应力使基体单元开裂. 孔隙周边基体单元的破坏及相互贯通主要是由于 基体单元的横向拉应力或拉应变超过材料的极限值. 模拟得到的孔隙介质的应力波传播规律、 变形与破坏模式以及能量耗散性质与物理模型的实验结果相吻合. 本文研究为解析岩石类孔隙 介质的复杂多变动力学响应的内在机制、应力传递模式、变形破坏与致灾机理提供了参考.
关键词

孔隙介质

三维模型

岩石

应力波

破坏机制

能量耗散

\section{1 引言}

近些年来我国煤矿重特大安全事故频发, 造成 了严重的人员和物质财产损失, 引起了全社会的广 泛关注. 除人为因素外, 矿山灾害孕育、发生和演化 的复杂性以及致灾机理认识不清是导致灾害事故逐 年上升和难以有效预测和预警的重要原因之一. 我 国大型煤矿普遍采用大规模集约化开采技术，高应
力、强卸荷和反复扰动导致围岩应力场剧烈变化, 矿 震、冲击地压、大面积顶板来压、煤与瓦斯突出等动 力灾害问题十分突出. 与此同时, 为了减轻和防治动 力灾害, 危险矿区多采取煤层卸载爆破、深孔断顶爆 破等解危方法来试图改善岩体介质性质, 缓解高应 力集中和减弱岩体弹性能的积聚程度, 但是人们对 于爆炸应力波对岩体结构的影响和诱发继生灾害(如 突水、煤瓦斯突出、矿震和岩爆等)的机制认识不充 
分，导致继生灾害难以有效控制，严重威胁矿山安全 生产. 揭示动力灾害的成灾过程、时空演化规律和致 灾机理已成为构建煤矿灾害事故监测预警体系、实现 煤矿安全生产的紧迫课题之一.

国内外研究表明: 矿山动力灾害是特定地质赋 存条件和开采扰动下煤岩体系统能量积聚和释放的 非线性动力学过程，是地质环境、地质构造、地应力、 岩体的结构和物理力学性质综合作用的结果, 其形 成过程复杂、影响因素众多 ${ }^{[1 \sim 2]}$. 揭示矿山动力灾害 的成灾过程和致灾机理涉及众多科学难题, 其中, 强 开采扰动或爆破施工产生的应力波在岩体中的传播 规律和能量积聚耗散特征是一个基础和关键科学问 题，该问题的解决对于揭示岩体(特别是富含孔隙或 裂隙岩体)中高应力的传递规律、能量积聚与释放机 制、动力灾变的致灾机理和触发条件具有重要意义.

矿山工程中的岩石或岩体原始存在着大量几何 不规则、跨尺度分布的孔隙或孔洞(例如煤系地层中 含气或含水的石灰岩, 顶底板砂岩, 白云岩, 凝灰岩 和玄武岩等), 孔隙率约在 5\% 35\%之间不等. 从理 论上讲，孔隙结构(或孔隙空间)的几何形态、尺寸、 分布、连通性以及变形特征会显著影响孔隙岩石或岩 体的应力传递、能量积聚与耗散以及整体失稳破坏 (灾变)行为, 由于孔隙数量众多、空间形态复杂且无 序分布, 从理论上给出岩石或岩体的应力波动特征、 本构方程以及能量积聚耗散随岩性、孔隙结构特征和 变形性质变化的定量关系极其困难 ${ }^{[13 \sim 20]}$, 人们更多地 依赖实验室和现场实验以及计算机模拟等手段来分析 应力波在天然孔隙岩石或岩体中的传播规律 ${ }^{[21 ~ 32]}$, 这 些研究为帮助人们理解和刻画孔隙岩石或岩体的高 应力传播与衰减规律、变形破坏机制以及动力灾害的 触发机制发挥了重要作用.

然而, 由于问题的复杂性和实验手段的限制, 目 前相关研究多集中在应力波动的表观特征及其随孔 隙岩石或岩体宏观物理力学参数 (如孔隙率, 孔隙压 力, 固相介质的强度、密度、弹性模量、泊松比以及 气液相介质性质)变化等方面, 很少能直观地显示或 刻画波动应力在岩石孔隙结构中的传递过程、能量积 聚耗散特征以及孔隙结构的变形破坏行为, 因而难 以科学地阐述波动应力作用下孔隙岩石或岩体动力 响应复杂多变和结构整体失效(灾变)的内在机理. 天 然岩石就像一个 “黑箱”, 大多数情况下人们只能观 测应力波动过程中 “黑箱”的表观力学特性, 却难以
获知和定量描述 “黑箱” 内部孔隙结构的变化及其作 用机制. 值得注意的是, 近些年来，一些研究者尝试 利用数值模拟和 CT 扫描实验等方法来分析孔隙结构 或孔隙空间特征对岩石类孔隙介质中应力传递与波 动规律的影响, 取得了初步的成效 ${ }^{[16,21,25 ~ 29,33]}$, 但距 离揭示波动应力作用下岩石“黑箱”的内部孔隙结构 效应仍有相当大的距离.

本文尝试通过构建岩石类孔隙介质的三维数值 模型，利用有限元方法模拟和分析冲击应力波作用 下岩石类孔隙介质的应力波动特征与变形破坏机制, 并与 SHPB 冲击试验和 CT 扫描试验结果进行对比, 为揭示孔隙岩石复杂多变的动力学响应与失效破坏 的内在机理提供途径和基础.

众所周知，由于自然沉积作用，天然孔隙岩石或 岩体的组成十分复杂，即使采样尺度相当小，岩石固 相和气液相的物理力学性质、孔隙结构的形态、尺寸 和分布也会有较大的差别, 很难获得除孔隙结构特 征不同之外其他物性参数相同的岩石或岩体样本. 因此，为了区分和识别孔隙结构特征对波动应力传播 性质的影响，并便于实验验证，本文采取以下方法： (1)首先通过 CT 扫描实验从孔隙率 22\% 23\%的天然 砂岩中提取孔隙特征参数, 通过 $\mathrm{X}$ 射线衍射和物理 实验获取砂岩样品的矿物成分和物理力学性质; (2) 利用模型材料加工制作具有与天然砂岩相同孔隙统 计特征、相同基体性质、不同孔隙率的孔隙介质模型 来模拟孔隙岩石; (3)利用孔隙介质物理模型的 CT 扫 描图像和软件 Mimics ${ }^{\circledR}$ 构建不同孔隙率的孔隙结构 三维有限单元模型; (4)将孔隙介质有限元模型导入 DYNA 程序中, 通过模拟 SHPB 冲击过程来分析冲击 应力波作用下孔隙介质的动力学响应、应力传递以及 孔隙结构的变形破坏过程, 探讨孔隙结构特征对应 力波动性质的影响.

需要指出的是, 除了能够直观定量地显示孔隙 结构的应力传递与变形破坏过程外, 采用数值模拟 方法的另一个重要原因是考虑到动力冲击实验 (如 SHPB 实验)很难完全消除一些显著影响岩石应力波 动行为的因素, 并满足动力学理论解条件和假设. 例 如，应力波传播过程中的高应变率效应，弥散效应以 及接触面的摩擦效应等 ${ }^{[34 ~ 38]}$, 而数值模拟可以较好 地解决这些问题, 数值模拟与实验观测相结合可以 更全面、准确地认识孔隙岩石的应力波动性质. 


\section{2 砂岩孔隙特征参数的识取}

图 1 为实验用砂岩样品, 取自我国西南地区. 样 品为 $\Phi 50 \mathrm{~mm} \times 100 \mathrm{~mm}$ 圆柱体. 图 2 是砂岩孔隙结构 及周围石英晶粒和胶结物状态的 SEM 照片 (放大 1000 倍). X 射线衍射和 SEM 微观分析表明: 砂岩样 品矿物成分为石英 $72.2 \%$, 钾长石 $9.7 \%$, 钠长石 $9.5 \%$, 粘土矿物 $8.6 \%$, 矿物成分空间分布均匀. 孔隙 形状接近圆球形，实测孔隙率在 $22.9 \%$ 23.8\% 之间不 等. 干燥条件下样品单轴静抗压强度平均值约为 $37.2 \mathrm{MPa}$, 弹性模量平均值约为 $4.32 \mathrm{GPa}$. 为了提取 孔隙结构特征, 对每个样品中部 $1 / 3$ 高度范围内自上 而下间隔 $80 \mu \mathrm{m}$ 进行 CT 扫描, 连续扫描 200 层, 得 到一组像素 $512 \times 512$ 的横截面灰度图. 利用文献[33] 方法得到各扫描层内孔隙数目沿周向的概率分布、孔 隙间距的概率分布和孔隙尺寸(即孔径)的概率分布, 如图 3 5 所示. 重复扫描 4 个样品, 得到上述孔隙特 征的统计分布函数如下.

孔隙数沿周向分布的概率密度函数:

$$
f(x)=0.0515-0.0000189 x,
$$

式中, $f(x)$ 表示周向分布孔隙数的概率密度函数, $x$ 为

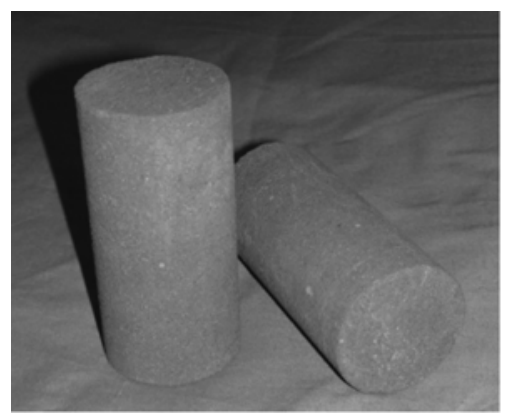

图 1 砂岩样品实物照片

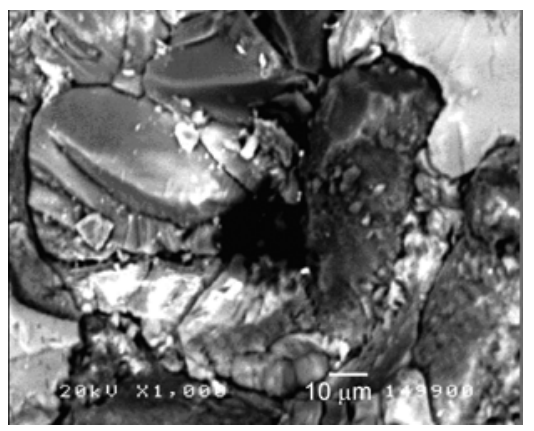

图 2 砂岩孔隙结构、石英颗粒与胶结物的 SEM 照片 (×1000 倍)

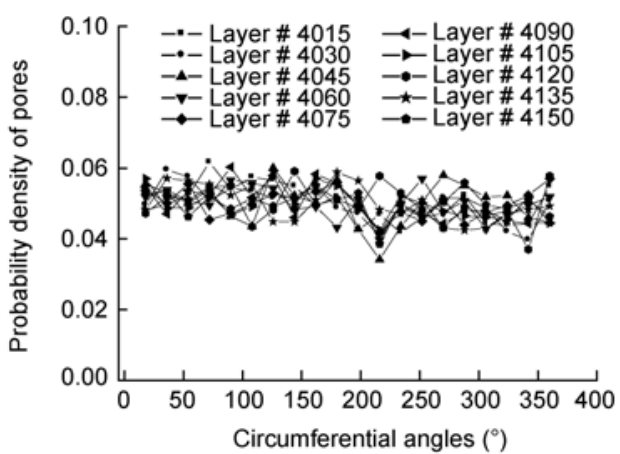

图 3 ４ 样品代表层孔隙数沿周向的概率密度分布

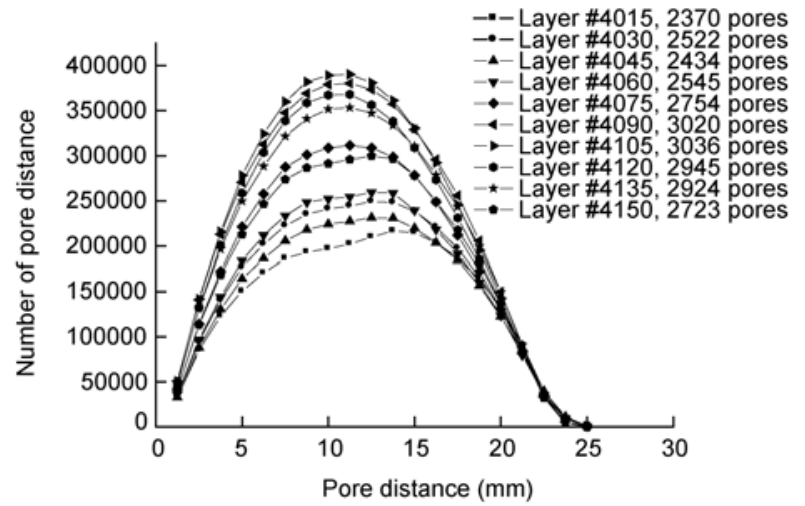

图 4 ＃4 样品代表层孔隙间距的累积概率分布

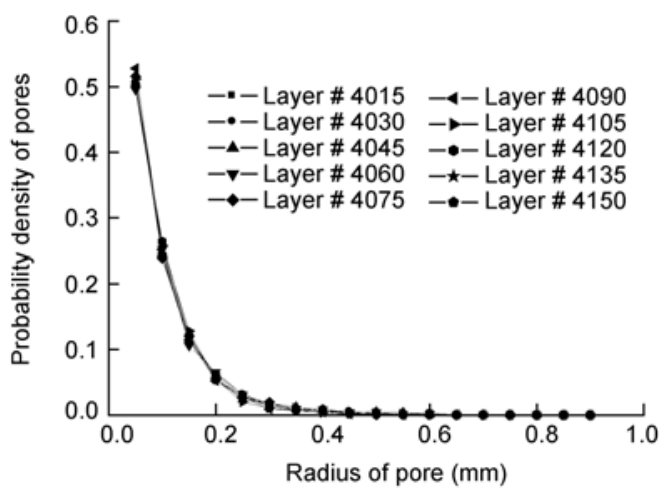

图 5 \#4 样品代表层孔隙孔径的概率密度分布

随机孔隙数. 公式右边第二项系数很小, 可忽略不计, 表明孔隙数沿周向呈均匀分布.

孔隙间距的概率密度函数:

$$
y=y_{0}+\left(\frac{A}{B \sqrt{\frac{\pi}{2}}}\right) \mathrm{e}^{-2\left(\frac{L-L_{0}}{B}\right)^{2}},
$$

式中, $y_{0}, L_{0}, A$ 和 $B$ 为孔间距分布的统计参数. 实测表 
明, 这些统计参数随砂岩各层孔隙数量呈线性变化 ${ }^{[33]}$, 可近似采用孔隙数 $N$ 表示为

$$
y_{0}=-525763.4+213.2 \mathrm{~N}, \quad L_{0}=15.4-0.002 \mathrm{~N},
$$

$A=1.984 \times 10^{7}-6791 N, B=54.74+0.0183 N$. 代入公 式(2)得到孔隙间距的概率分布函数.

孔隙孔径 $r$ 的概率密度函数 $g(r)$ :

$$
g(r)=C \times \exp \left(-\frac{r}{D}\right)+E,
$$

式中, $C, D$ 和 $E$ 为孔径分布统计参数.

\section{3 孔隙介质的物理模型}

为了模拟孔隙岩石，并识别孔隙特征对介质应 力波动行为的影响, 采用水泥砂浆和聚苯乙烯颗粒 来分别模拟岩石固体相和孔隙相. 用石英砂作为粗 骨料, 经适当方法成型后, 孔隙结构模型的均匀和密 实程度与砂岩相当. 表 1 列出了孔隙模型的材料组成 与配比. 模拟孔隙的聚苯乙烯颗粒质量轻、强度低、 内有空气层, 与天然砂岩孔隙相的物理力学性质十分 相近. 根据实测砂岩孔径的概率分布规律(见公式(3)), 将聚苯乙烯颗粒按直径大小分成 6 个等级, 即 $1.8,2.3$, 2.6, 3.3, 4 和 $5.2 \mathrm{~mm}$. 再根据设计孔隙率 $\rho_{v}$ 和孔隙数量 的分布规律(见公式(1))确定出每种孔隙率模型所需要 的不同粒径的聚苯乙烯颗粒数目. 本文设计了 5 种孔 隙率模型来考察孔隙数量对介质波动性质的影响, 孔 隙率 $\rho_{v}$ 分别为 $25 \%, 20 \%, 15 \%, 10 \%$ 和 $5 \%$.

制作孔隙物理模型时, 先将称量好的石英砂和 水泥混合搅拌 $3 \mathrm{~min}$, 再掺入额定数量的聚苯乙烯颗
粒摚拌 $3 \mathrm{~min}$, 然后加入 $1 / 2$ 融合好的水和减水剂摚 拌 $3 \mathrm{~min}$, 再倒入剩余的 $1 / 2$ 水和减水剂搅拌至均匀. 将拌合料浇注于模具中, 振捣成型后 $24 \mathrm{~h}$ 脱模. 脱模 后的试件放入高温养护箱中 $90^{\circ} \mathrm{C}$ 养护 $72 \mathrm{~h}$. 模型为 $\Phi 71 \mathrm{~mm} \times 30 \mathrm{~mm}$ 的圆盘试件(该尺寸系按 SHPB 冲击 实验要求确定 ${ }^{[39,40]}$. 所有模型经过相同的配比、加工 方式和养护方法制作而成. 相同孔隙率的物理模型 一次制作完成, 以保证孔隙模型基体的物理力学性 质相一致和实验结果的可比性.

为了检验物理模型与天然砂岩孔隙结构的相似 程度, 本文对孔隙物理模型进行了 CT 扫描. 考虑到 聚苯乙烯颗粒的最小尺寸, 每种孔隙率模型沿高度 方向间隔 $0.5 \mathrm{~mm}$ 扫描横截面, 获得 60 个横断面扫描 层, 这样可以保证 $\mathrm{CT}$ 扫描没有遗漏孔隙. 图 6 给出 了 5 种孔隙率模型代表层(即第 25 层横断面)的 CT 图 像，图中黑点为孔隙(即聚苯乙烯颗粒).

考虑到孔隙的尺寸是依据天然砂岩孔径分布函 数(公式(3)) 确定的, 因此本文利用文献[33]的方法提 取了物理模型中孔隙数量和间距的统计特征. 图 7 和 8 分别绘出了 5 种孔隙率模型代表层中的孔隙数沿周 向的概率分布和孔隙间距的概率分布规律.

统计分析表明：(1)不同孔隙率物理模型的各扫 描层孔隙数的周向分布满足均匀分布, 其中孔隙率 $\rho_{v} \leq 10 \%$ 时, 孔隙数的周向分布具有一定离散性. 孔 隙率 $\rho_{v} \geq 15 \%$ 时, 孔隙数的周向分布较好地服从均匀 分布；2模型孔隙间距满足高斯分布，其中孔隙率 $\rho_{v}=5 \%$ 时孔隙间距的分布数据具有一定离散性, 其他 孔隙率条件下模型孔隙间距均较好地符合高斯分布.

\section{表 1 物理模型的材料组成与配比 $\left(\mathbf{m}^{3}\right)$}

\begin{tabular}{ccccc}
\hline Water cement ratio & Cement $(\mathrm{kg})$ & Quartz sand $(\mathrm{kg})$ & Super plasticizer $(\mathrm{L})$ & Water $(\mathrm{L})$ \\
\hline 0.2 & 991 & 1110 & 24.7 & 181.5 \\
\hline
\end{tabular}
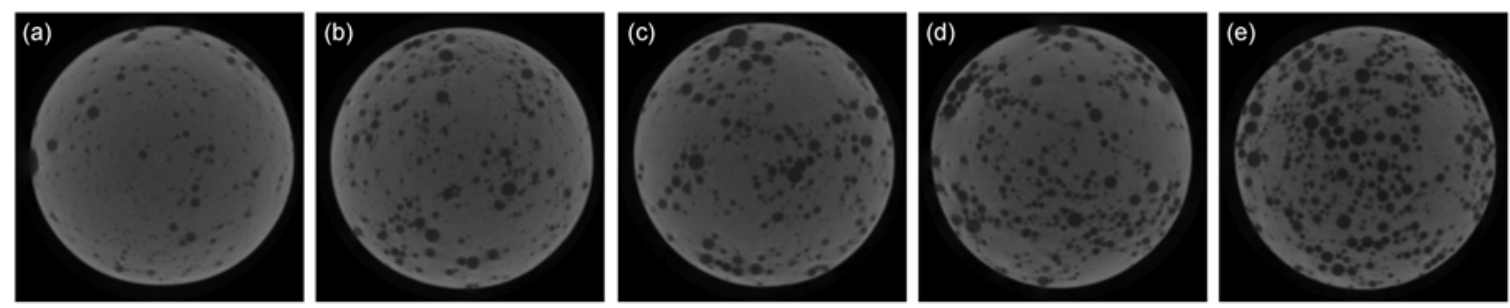

图 6 五种孔隙介质模型代表层中的孔隙形态与分布的 CT 图像(第 25 层断面)

(a) $\rho_{v}=5 \%$; (b) $\rho_{v}=10 \%$; (c) $\rho_{v}=15 \%$; (d) $\rho_{v}=20 \%$; (e) $\rho_{v}=25 \%$ 

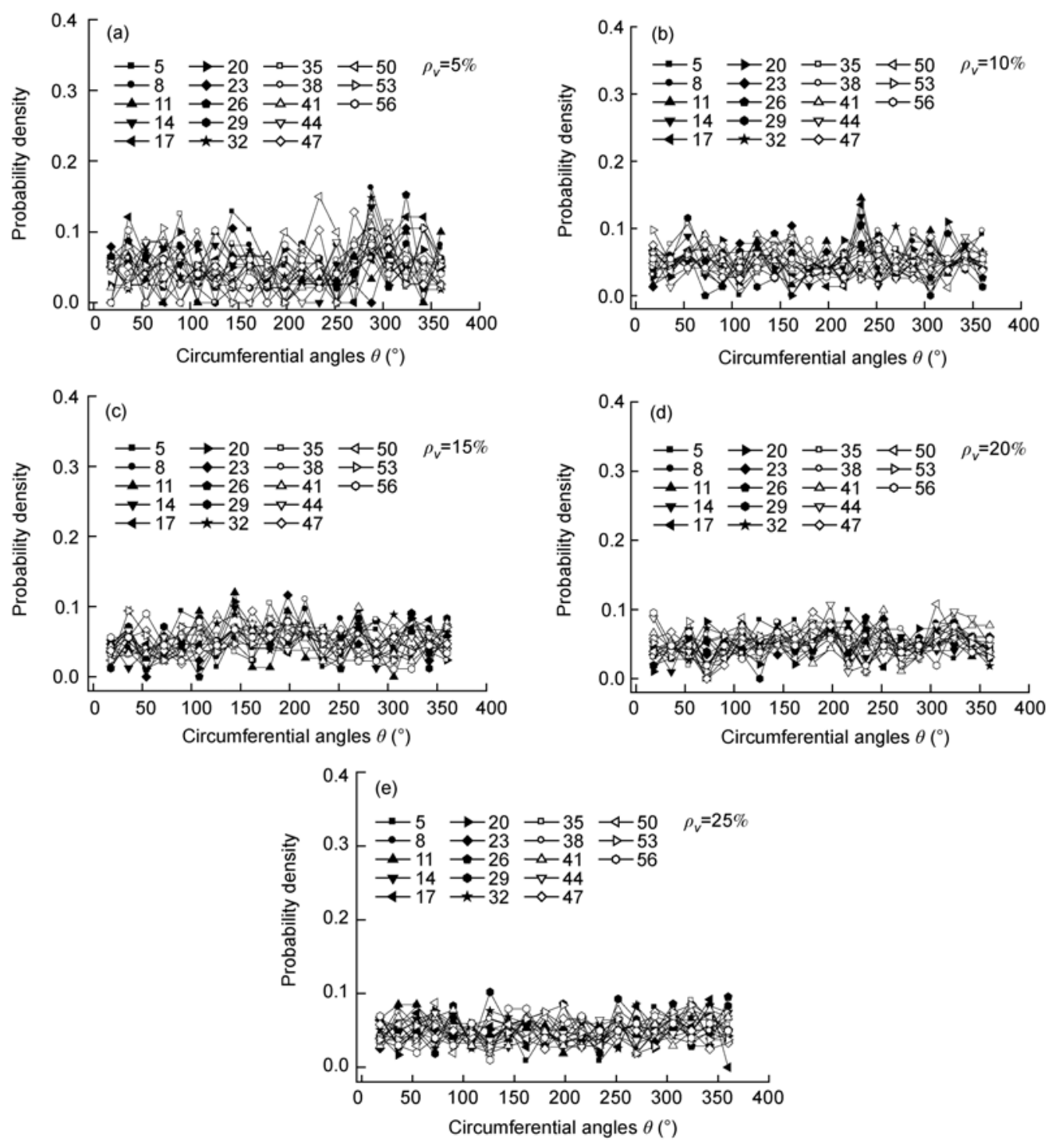

图 7 五种孔隙介质模型代表层中孔隙数沿周向的概率分布(图注中的数字表示扫描层的编号)

(a) $\rho_{v}=5 \%$; (b) $\rho_{v}=10 \%$; (c) $\rho_{v}=15 \%$; (d) $\rho_{v}=20 \%$; (e) $\rho_{v}=25 \%$

数据拟合表明: 物理模型孔隙数的周向分布和孔隙 间距分布的概率密度函数满足公式(1)和(2).

为了检验孔隙介质的物理力学性质, 进行了孔 隙介质不同尺寸圆柱体的轴心抗压试验，测试了其 轴心抗压强度和弹性模量. 受压试件的材料配比和 制作工艺完全相同, 2 种尺寸分别为 $\Phi 71 \mathrm{~mm} \times 143 \mathrm{~mm}$ 和 $\Phi 50 \mathrm{~mm} \times 100 \mathrm{~mm}$. 图 9 和 10 分别绘出了实测轴心 抗压强度和弹性模量随介质孔隙率的变化. 结果表 明, 孔隙介质具有与砂岩一致的抗压强度和弹性模 量变化规律 ${ }^{[39,40]}$.
实验结果表明: 孔隙介质物理模型在孔隙数目、 间距和尺寸的统计分布规律以及物理力学性质等方 面与天然砂岩保持一致, 这为利用物理模型和数值 算法分析孔隙结构特征对应力波动以及能量耗散机 制的影响奠定了基础.

\section{4 孔隙介质的三维有限元模型}

孔隙介质物理力学行为数值分析中一个具有挑 战性的难题是如何恰当地构建单元网格模型并对不 

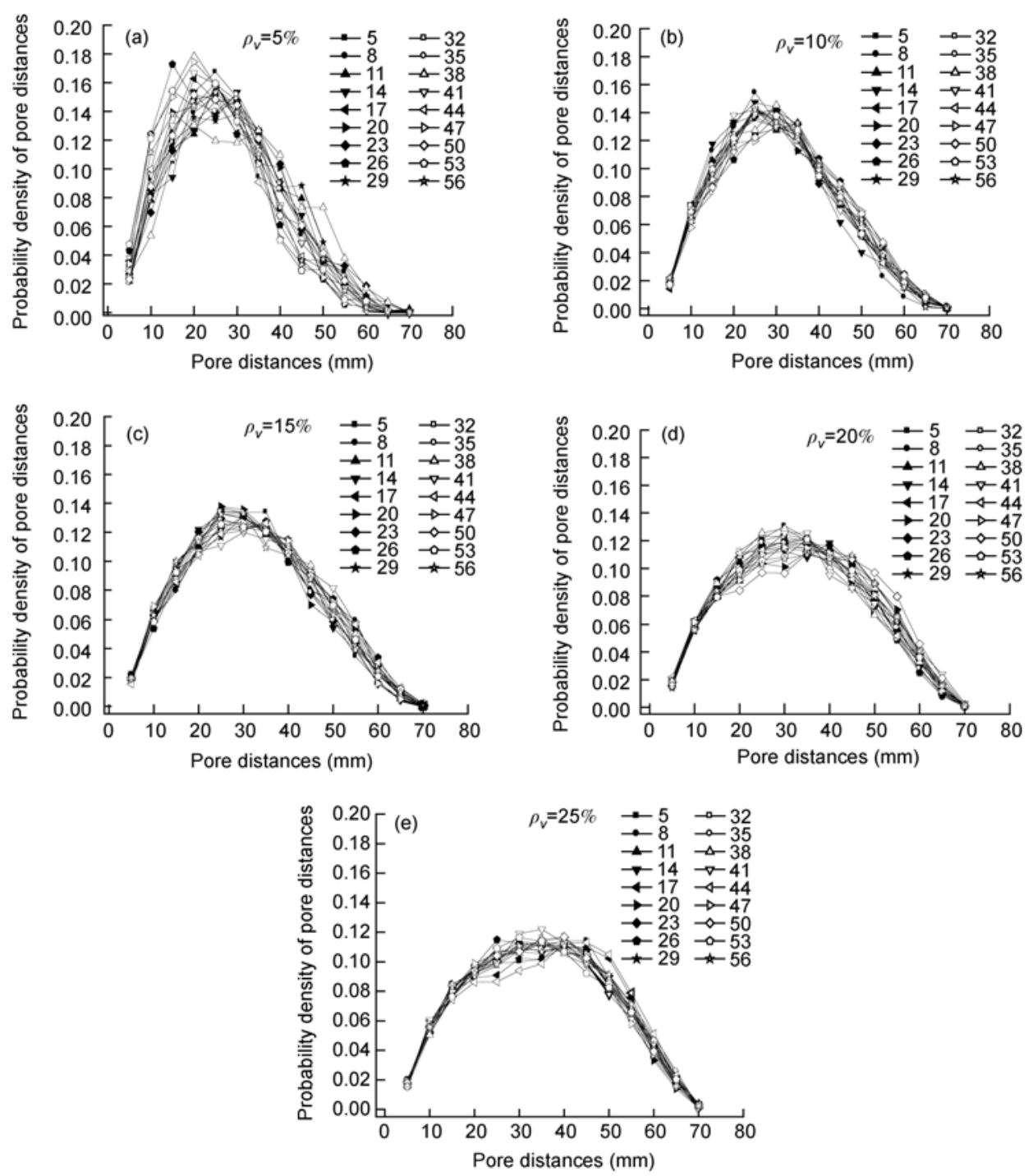

图 8 五种孔隙率模型中代表层孔隙间距的概率密度分布(图注中的数字表示扫描层的编号)

(a) $\rho_{v}=5 \%$; (b) $\rho_{v}=10 \%$; (c) $\rho_{v}=15 \%$; (d) $\rho_{v}=20 \%$; (e) $\rho_{v}=25 \%$

同介质相进行材料属性赋值, 它是进行介质应力与 变形分析的基础和关键. 由于岩石类介质中孔隙的 数量多、跨尺度分布和几何特性复杂等原因, 常规数 值算法(如有限元、边界元、离散元和有限差分等)在 建立网格模型时常遇到单元过多、网格畸变、孔隙与 基体界面难处理等问题, 导致数值计算常常难以进 行. 为克服上述困难, 本文采用基于 $\mathrm{CT}$ 图像的三维 重构程序 $\mathrm{MIMICS}^{\circledR}$ 来构建孔隙结构三维有限元模型. MIMICS ${ }^{\circledR}$ 是由 Materialise 公司开发的针对医学 CT 影 像进行三维重构与分析处理的软件, 具有识别断层 扫描数据(例如 CT, MRI)和三维重构与编辑处理的功 能. 它可以输出通用的 CAD, FEA 和 RP 数据格式,
从而实现大规模的数据转换与计算.

本文采取以下方法来构建孔隙介质的三维有限 元模型.

(1) 首先利用 $\mathrm{MIMICS}{ }^{\circledR}$ 读取孔隙物理模型的 二维 CT 图像(每个模型 60 个扫描层), 生成三维实体 模型，然后对实体模型和内部孔隙生成面网格。面 网格是由包裹实体模型和孔隙边界的三角片转化而 成. 为了克服初始生成的面网格单元数量多、质量差 等问题，采用控制几何误差来减少单元数量的方法 (如 REDUCE 命令)对初始面网格进行优化，然后利用 程序的“自动查找修复”工具修复初级网格优化中产 生的嵌入三角网格(insert triangles)和重复三角网格 


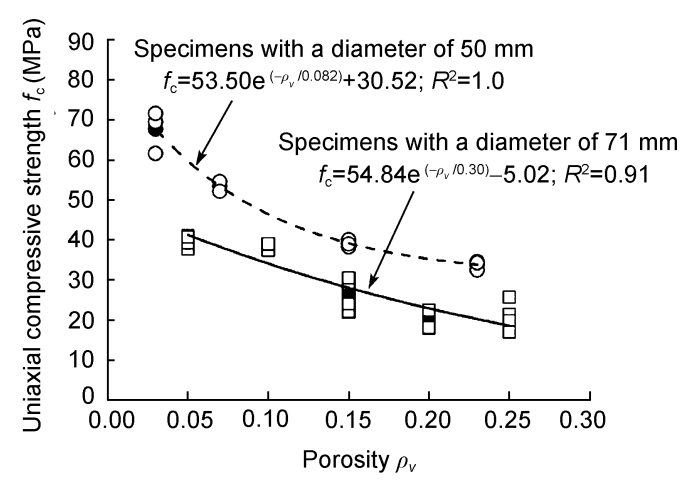

图 9 圆柱形孔隙试件 $\Phi 71 \mathrm{~mm} \times 143 \mathrm{~mm}$ 和 $\Phi 50 \mathrm{~mm} \times 100 \mathrm{~mm}$ 的轴心抗压强度随孔隙率变化的关系 图中空心点表示同种孔隙率强度的实测值, 实心点表示同种孔隙率 实测强度的平均值, 实线和虚线代表不同尺寸试件强度的拟合结果

(double triangles), 完成面网格优化.

(2) 考虑到计算精度和波动应力传递与变形分析 的需要, 对孔隙临近区域和密集区域进行了单元网 格加密和局部细化, 并对网格进一步优化.

(3) 在优化面网格的基础上, 利用映射算法生成 4 节点四面体单元网格. 体网格生成过程中可通过控 制网格尺寸来控制四面体单元网格的数量和质量.

(4) 对划分好的体网格进行材料属性赋值和确定 单元类型. MIMICS ${ }^{\circledR}$ 利用 $\mathrm{CT}$ 图像的灰度值进行材料 属性赋值. 理论上讲, 不同属性介质 CT 图像的灰度 值不同，可以通过设定灰度值范围对不同介质赋予 不同的材料参数. 考虑到本文所模拟的砂岩均质性较

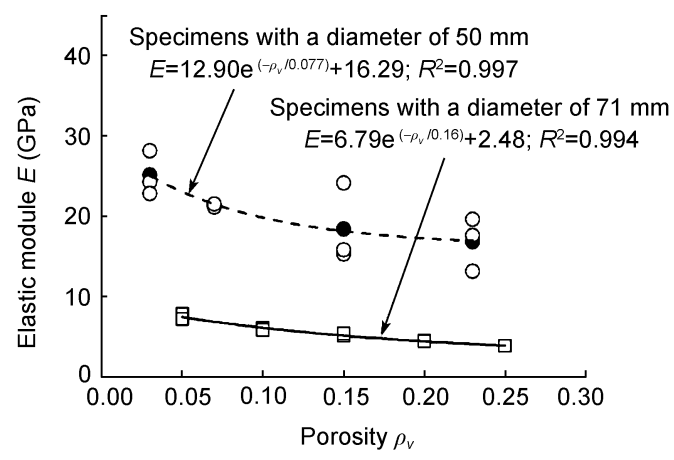

图 10 圆柱形孔隙试件 $\Phi 71 \mathrm{~mm} \times 143 \mathrm{~mm}$ 和 $\Phi 50 \mathrm{~mm}$

$\times 100 \mathrm{~mm}$ 轴心受压时弹性模量随孔隙率变化的关系

图中空心点表示同种孔隙率弹性模量的实测值, 实心点表示同种孔 隙率实测模量的平均值. 实线和虚线代表不同尺寸试件弹性模量的 拟合结果

好，矿物成分空间分布与固相物性的差别较小(砂岩的 $X$ 射线衍射、SEM 微观分析以及 CT 扫描试验 ${ }^{[33]}$ 均证 实了这一点), 因此对固体单元赋予同种材料属性. 根据不同孔隙率模型的单轴抗压实验结果, 取介质 孔隙率 $\rho_{v}=0$ 的单轴抗压强度 $\sigma_{u}$ 和弹性模量 $E$ 作为基体 的抗压强度和弹性模量, 即 $\sigma_{u}=70 \mathrm{MPa}, E=30 \mathrm{GPa}$. 取 基体单轴抗拉强度为 $\sigma_{t}=7 \mathrm{MPa}$. 考虑到 DYNA 模拟应 力波动过程时对单元类型的要求, 本文选取了 MESH200 单元, 并将 4 节点四面体单元转换为 10 节 点四面体单元.

图 11 为利用上述方法构建的不同孔隙率的孔隙介 质三维有限元模型, 尺寸为 $\Phi 71 \mathrm{~mm} \times 30 \mathrm{~mm}$. 图 12 以
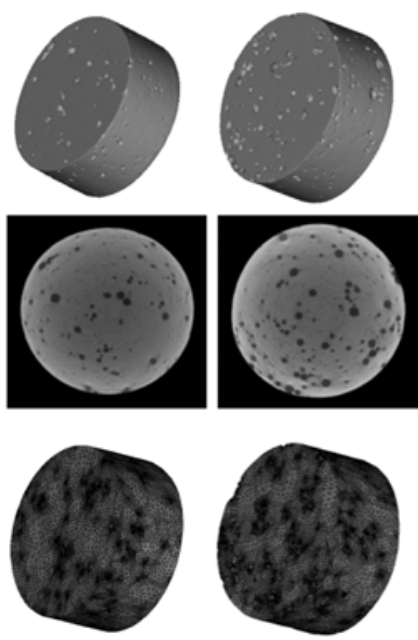
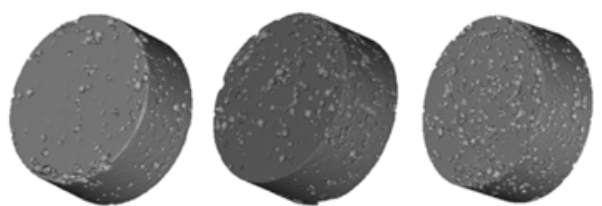

(a)
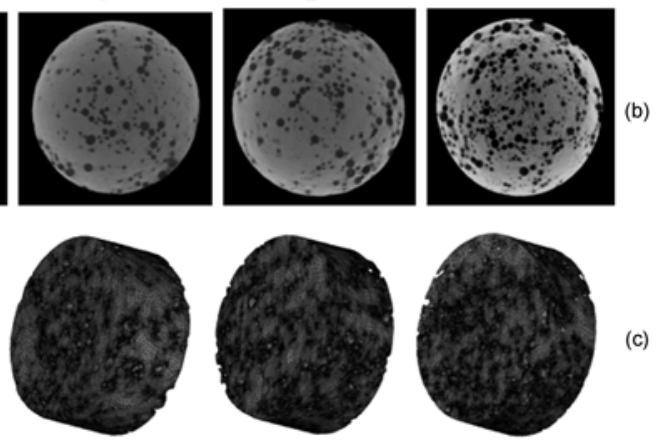

图 11 不同孔隙率孔隙介质的三维有限单元模型

(a) 重构的三维孔隙实体模型; (b) 实体模型 1/2 高度处的横截面图; (c) 三维实体单元网格模型.

从左至右介质孔隙率 $\rho_{v}$ 分别为 $5 \%, 10 \%, 15 \%, 20 \%$ 和 $25 \%$ 
孔隙率 $10 \%$ 的介质模型为例, 利用横截面和纵向剖 面图，显示了模型体单元以及孔隙周围单元网格的 细化情况.

建模结果表明: 利用 CT 图像和 MIMICS ${ }^{\circledR}$ 构建 的孔隙介质三维数值模型与物理模型具有很好的几 何一致性. 经过单元网格划分和优化后，模型的计算 孔隙率与物理模型的孔隙率一致. 孔隙三维数值模 型的体网格和面网格过渡均匀, 没有网格畸变.

\section{5 冲击过程模拟与动态响应分析}

分离式霍普金森压杆(SHPB， split Hopkinson pressure bar)主要用来测试固体介质的动态压缩性能. 将上述孔隙介质置于 SHPB 系统中, 利用具有一定初 速度的冲击锤撞击入射杆产生应力波，可以分析冲击 应力波作用下孔隙介质的动态响应性能. SHPB 装置 与加载方式如图 13 所示. 值得注意的是, 由于 SHPB 实验难以完全消除应力波传播时的二维弥散效应和接 触面的摩擦效应, 并满足一维波动理论假设 ${ }^{[21,34 ~ 38]}$, 岩石应力波动的实验与理论结果之间常存在较大偏差. 鉴于此, 我们利用孔隙介质三维数值模型和非线性有 限元程序 DYNA, 通过设定波动理论假设的条件来模 拟岩石类孔隙介质的 SHPB 冲击过程. 通过数值分析
获得压杆入射波、反射波和透射波性质，按波动理论 分析和计算不同孔隙率介质的内部应力、变形与能量 耗散。

\section{1 计算模型}

图 14 以孔隙率 $15 \%$ 试件为例, 给出了 SHPB 冲 击的有限元计算模型. 其中, 孔隙试件长 $30 \mathrm{~mm}$, 直 径 $71 \mathrm{~mm}$, 该尺寸按照 SHPB 冲击实验要求和一维波 动理论确定 ${ }^{[39,40]}$ (物理模型的 SHPB 冲击实验作者另 文介绍). 为了与物理实验结果对比, 模拟时根据 SHPB 装置的实际尺寸，取入射杆和透射杆的直径为 $72 \mathrm{~mm}$ ，长 $1800 \mathrm{~mm}$. 入射波采用三角形脉冲波，周 期 $0.3 \mathrm{~ms}$, 波长 $1.56 \mathrm{~m}$, 波速 $v_{0}=5200 \mathrm{~m} / \mathrm{s}$, 峰值压应 力 $120 \mathrm{MPa}$ (相当于实际冲击速度 $16.9 \mathrm{~m} / \mathrm{s}$, 应变率 $\dot{\varepsilon} \leq 90 / \mathrm{s}$ ). 杆身采用钢材模拟, 参数取值: 密度 $\rho_{0}=7850 \mathrm{~kg} / \mathrm{m}^{3}$, 弹性模量 $E=214 \mathrm{GPa}$, 泊松比 $v=0.26$. 杆件采用 8 节点 Solid164 单元.

为了反映冲击荷载作用下孔隙介质可能发生的非 线性变形破坏，根据天然砂岩以及孔隙物理模型的冲 击试验结果 ${ }^{[21,39]}$, 本文采用 $\mathrm{HJC}$ (Holmquist-JohnsonCook)本构模型来描述固相介质的非线性特征，材料参 数取值如表 2 所示. 单元破坏准则选用最大拉应力准则. 为方便计算，将孔隙介质三维有限元模型 MESH200

图 12 孔隙结构模型的体单元网格与局部细节(孔隙率 $\rho_{v}=10 \%$ ) 


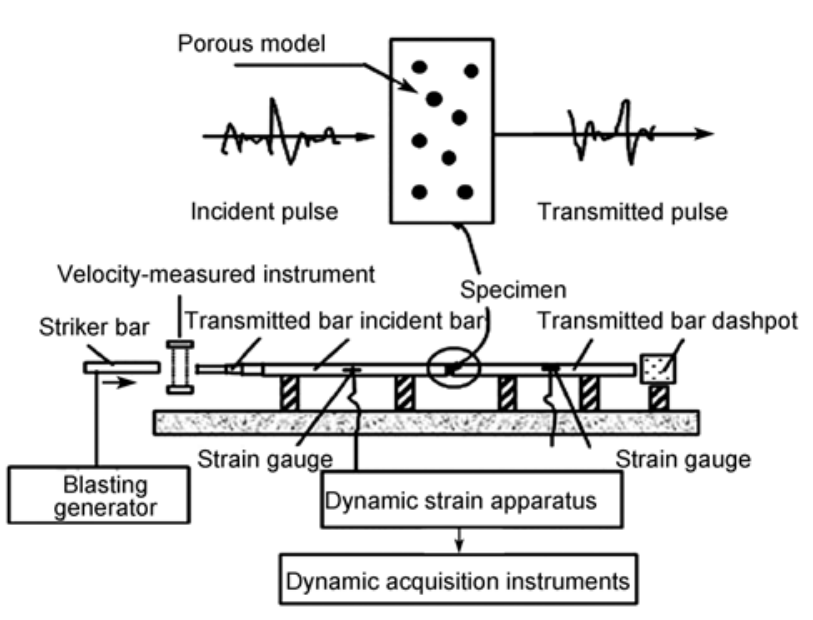

图 13 SHPB 装置和冲击加载方案示意图

图 14 SHPB 冲击过程的有限元计算模型(孔隙率 $\rho_{v}=15 \%$ )

单元转换为 DYNA 中同类型的 10 节点四面体单元 Solid168.

孔隙模型与入射杆和透射杆的接触采用 SURFACETO-SURFAC 自动接触模型. 由于孔隙随机分布的影 响, 孔隙模型接触面局部会出现凹凸不平, 影响杆中 应力的分布与传递规律. 为有效地模拟应力波传播, 采用局部节点坐标修正使接触面平滑的方法来模拟 实际样品表面与杆件的接触状态. 平行接触面方向 设为自由边界, 摩擦系数为零, 以消除接触面摩擦效 应的影响. 同时, 为了消除应力波传播的二维弥散效 应, 满足一维应力波理论假定, 入射杆和透射杆上所 有节点沿 $X$ 和 $Y$ 方向设置位移约束, 应力波只沿 $Z$ 方
向振动和传播. 为了反映冲击时杆端对应力波的吸 收作用，模型中入射杆和透射杆的远端设为无反射 边界.

\section{2 计算结果与分析}

图 15 以冲击速度 $16.9 \mathrm{~m} / \mathrm{s}$ (应变率 $\dot{\varepsilon} \leq 90 / \mathrm{s}$ )、孔 隙率 $15 \%$ 的试件为例，绘出了压缩波在入射杆、孔隙 介质和透射杆中的传播过程, 通过局部放大显示了 应力波穿越孔隙介质时接触面左右两侧杆中应力和 孔隙介质应力的变化. 为了说明数值模拟的有效性 以及孔隙对介质应力波动与传输性质的影响, 图 16 给出了入射杆和透射杆长 $1 / 2$ 位置处的应力波形随介 质孔隙率变化的模拟结果和物理模型实验结果 ${ }^{[39]}$. 图 17 给出了图 15 的不同波动时刻下孔隙介质单元正 应力 (z-轴方向, 即波传播方向)和最大剪应力的分布 与变化, 图 18 为对应时刻下孔隙介质单元应变的计算 结果. 作为对比, 图 19 给出了孔隙介质物理模型冲击 后内部结构变形破坏的 CT 图像.

模拟结果清楚地显示:

(i) 压杆中以及孔隙介质与压杆交界面处的应力 波传播、透射和反射的数值模拟结果与物理实验结果 具有很好的一致性. 入射波在孔隙试件与压杆交界 面处产生明显反射，反射波在入射杆中与入射波相 互叠加(见图 15). 相同冲击速度下, 随着介质孔隙率 增大，反射波幅增加，透射波幅减小(见图 16). 单元 的正应力、最大剪应力和变形计算结果表明: 高应力 单元和由于拉应力或拉应变而破坏的单元(即开裂) 主要分布在圆柱形试件横截面外缘区域(见图 17I(f), II(d), II(e); 18I(e), 18II(e)). 这些计算结果与物理模型 观测结果相吻合(见图 19). 表明本文构建的孔隙介质 三维模型和应力波动过程的有限元计算模型有效. 需 要指出的是, 由于实际实验条件和数值模拟条件的 差异，模拟得到的入射波和反射波形与实验实测波 形局部存在偏差.

\section{表 2 孔隙介质固体相 HJC 本构模型的参数}

\begin{tabular}{cccccccccc}
\hline$\rho\left(\mathrm{kg} / \mathrm{mm}^{3}\right)$ & $F_{\mathrm{C}}(\mathrm{GPa})$ & $A$ & $B$ & $C$ & SFMAX & $G(\mathrm{GPa})$ & $D_{1}$ & $D_{2}$ & $N$ \\
\hline \hline $2.4 \times 10^{-6}$ & 0.07 & 0.79 & 1.6 & 0.007 & 7 & 15 & 0.04 & 1 & 0.61 \\
\hline \hline & & & & & & & & & \\
EFMIN & $T(\mathrm{GPa})$ & $P_{\mathrm{C}}(\mathrm{GPa})$ & $\mu_{\mathrm{C}}$ & $P_{\mathrm{L}}(\mathrm{GPa})$ & $\mu_{\mathrm{L}}$ & $K_{1}(\mathrm{GPa})$ & $K_{2}(\mathrm{GPa})$ & $K_{3}(\mathrm{GPa})$ & $F_{\mathrm{S}}(\mathrm{GPa})$ \\
0.01 & 0.01 & 0.023 & 0.001 & 0.8 & 0.1 & 85 & -171 & 208 & -0.007 \\
\hline
\end{tabular}


图 15 应力波的传递过程与孔隙试件的应力变化图(孔隙率 15\%为例)

三角形应力波由左向右传播. 彩色条纹表示不同的应力水平，正号代表拉应力，负号代表压应力. (a) 应力波在入射杆中传递，波前尚未达到 孔隙试件; (b) 波前进入孔隙试件, 应力波在入射杆与试件交界面处产生反射; (c) 应力波前越过孔隙试件进入透射杆, 应力波在试件与入射 杆和透射杆交界面处产生反射; (d) 应力波峰作用于孔隙试件; (e) 应力波峰越过孔隙试件, 应力波继续在透射杆上延伸; (f) 应力波完全离开 孔隙试件, 在透射杆中传播

\section{图 16 入射杆和透射杆长 $1 / 2$ 位置处的应力波形随介质孔隙率的变化}

(a) 数值模拟结果; (b) 物理模型实验结果

(ii) 入射波作用下孔隙介质首先发生压缩变形, 当压缩波逐步离开孔隙体后，基体固相的弹性变形 恢复对入射杆产生一个反向的压缩应力，该压缩波 与入射杆和介质交界面处产生的反射拉伸波相互叠 加，导致入射杆上记录的反射波波长小于入射波波 长. 孔隙率越小, 固相占孔隙介质的比例越高, 可恢 复的弹性变形越大，因而反射波波幅越小，波长越短。 数值模拟与实验结果均证实了这一点(见图 16). (iii) 应力波进入孔隙试件前，沿波传播方向的 基体单元正应力为均匀分布的压应力. 应力波进入 孔隙试件后，随着波幅增加，基体单元压应力增大， 但由于孔隙以及界面反射波的影响，应力分布不均 匀. 该阶段前基体单元最大剪应力分布较均匀(见图 17I(a), I(b); II(a), II(b)). 当应力波穿越孔隙试件进入 透射杆后，由于应力波幅增加，基体单元正应力持续 增加. 应力波在试件与压杆界面多次反射, 试件横截 
图 17 不同波动时刻下孔隙介质单元正应力和最大剪应力的分布与变化(孔隙率 $15 \%$ 为例)

每幅图包含三维体视图以及 $x-y, y-z$ 和 $z-x$ 方向截面视图. 图中应力标尺单位为 GPa. (a) 应力波在入射杆中传递, 波前尚未达到孔隙试件时; (b) 波前进入孔隙试件, 应力波在入射杆与试件交界面处产生反射时; (c) 应力波前越过孔隙试件进入透射杆, 应力波在试件与入射杆和透 射杆交界面处产生反射时; (d) 应力波峰作用于孔隙试件; (e) 应力波峰越过孔隙试件, 应力波继续在透射杆上延伸时; (f) 应力波完全离开孔 隙试件在透射杆中传播时 
图 18 不同波动时刻下孔隙介质单元沿 $\boldsymbol{x}$ 和 $\boldsymbol{y}$ 轴方向的应变(孔隙率 $15 \%$ 为例) 每幅图包含三维体视图以及 $x-y, y-z$ 和 $x-z$ 方向截面视图. (a) 应力波在入射杆中传递, 波前尚未达到孔隙试件 时；(b) 波前进入孔隙试件，应力波在入射杆与试件交界面处产生反射时; (c) 应力波穿越整个孔隙试件, 波前 进入透射杆, 应力波在试件与入射杆和透射杆交界面处产生反射时; (d) 应力波峰作用于孔隙试件; (e) 应力波 峰越过孔隙试件, 应力波继续在透射杆上延伸时; (f) 应力波完全离开孔隙试件在透射杆中传播时 

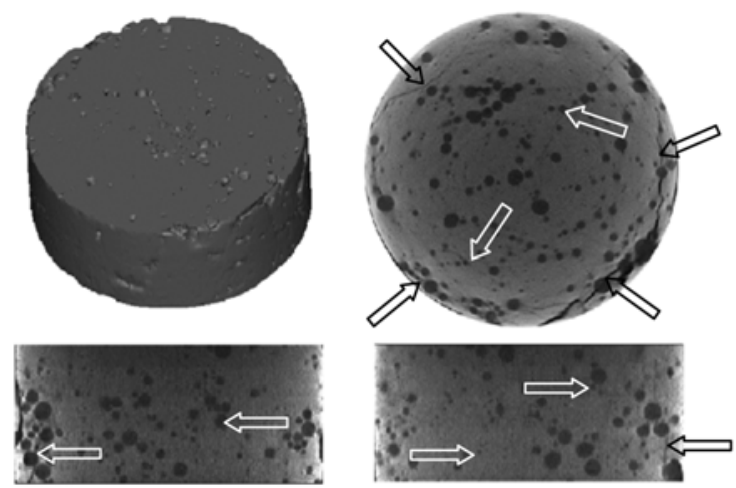

图 19 孔隙介质物理模型冲击后内部结构变形破坏的 CT 图像(孔隙率 15\%)

图中包含三维体视图以及 $x-y, y-z$ 和 $x-z$ 方向截面视图. 图中箭头 所指为肉眼可识别的基体开裂裂纹和破裂连通区域

面边缘和两端接触面上的部分孔隙周边单元出现较 大的拉应力, 孔隙周边单元最大剪应力增加, 剪应力 分布不均匀(见图 17I(c), II(c)). 当应力波峰到达孔隙 试件时, 由于压应力较大, 除横截面边缘少数单元仍 有较高的拉应力外, 基体大部分单元的正应力转为 压应力. 同时, 由于孔隙分布和反射波的影响, 试件 内部及两端接触面上的部分孔隙的周边单元最大剪 应力达到最大, 而且这种孔隙的数量较多 (见图 17I(d), II(d)). 当压应力波峰越过孔隙试件进入透射 杆后, 压应力幅值开始降低, 但仍保持较高的水平 (约为压应力峰值 $48 \%$ 52\%). 除横截面边缘少数孔 隙的周边单元受拉应力外, 基体大部分单元的正应 力仍为压应力. 与应力波峰值时刻相比, 试件内部及 两端接触面上孔隙周边单元的最大剪应力水平较高, 而且高剪应力基体单元的分布范围扩大(见图 17I(e), II(e)). 当应力波完全离开试件在透射杆中传播时, 孔隙试件中的压应力幅值降低至零. 由于基体的微 塑性(剪切变形)和开裂(横向拉应变)等不可逆变性以 及反向拉伸波的作用, 部分孔隙的周边单元同时出 现较高的拉应力和剪应力, 单元发生破坏, 且破坏的 单元相互贯通(见图 17I(f), II(f)), 表明孔隙试件在这 些部位产生了过大的不可逆变形.

(iv) 应力波穿越试件进入透射杆前, 孔隙周围 基体介质的横向变形(即沿 $x, y$ 轴方向应变)很小, 应 变范围 $-0.16 \% \leq \varepsilon_{x}, \quad \varepsilon_{y} \leq 0.08 \%$, 且主要为压应变 (见图 18I(a)，I(b); II(a)，II(b)). 当应力波进入透射杆后，由 于界面反射波和入射波的叠加, 圆柱形试件侧面和
两端接触面上少数孔隙的周边单元产生较大的横向 拉应变，范围为 $0.08 \% \leq \varepsilon_{x}, \varepsilon_{y} \leq 0.24 \%$. 该阶段由于压 应力幅值较高, 绝大部分基体单元的横向变形仍为 压应变(见图 18I(c), II(c)). 当压应力波峰到达孔隙试 件时，入射波、反射波的持续作用以及叠加效应使得 孔隙试件侧面和两端接触面上原孔隙周边单元的横 向拉应变进一步增大. 同时, 更多孔隙的邻域单元出 现了较大的横向拉应变. 该阶段由于入射波处于压 应力峰值, 反射波并未造成基体单元大范围出现横 向拉应变(见图 18I(d), II(d)). 当应力波峰值越过孔隙 试件进入透射杆后, 由于压应力幅值降低(压应力峰 值的 $48 \%$ 52\%), 在反射波和入射波叠加作用下，孔 隙试件的侧面、两端接触面以及内部横向变形较大的 单元产生更大的横向拉应变, 而且这类单元的数目 和分布范围进一步扩大, 并相互连通. 横向拉应变范 围为 $0.08 \% \leq \varepsilon_{x}, \varepsilon_{y} \leq 0.4 \%$ (见图 18I(e), II(e)). 当基体单 元的拉应变超过材料的极限拉应变时, 上述部位的单 元产生破裂. 当应力波完全离开孔隙试件在透射杆中 传播时, 入射波的压应力幅值降低至零, 反向拉伸波作 用也逐渐减弱, 基体弹性变形得到一定程度的释放, 部 分基体单元的变形恢复为压应变. 由于部分孔隙的周 边单元已经发生开裂或微塑性等不可逆变形, 因而这 部分孔隙的周边单元仍有较高的横向拉应变, 并相互 贯通(见图 18I(f), II(f)). 该结果与应力计算中发现的高 应力区和单元破裂区的分布以及物理模型 $\mathrm{CT}$ 实验观测 到的破裂区相吻合(见图 17 和 19).

上述分析表明：一定压强和波速的应力波传播过 程中(冲击速度 $v_{0} \leq 16.9 \mathrm{~m} / \mathrm{s}$, 应变率 $\dot{\varepsilon} \leq 90 / \mathrm{s}$ ), 孔隙率 $\rho_{v}=15 \%$ 的岩石类介质的内部孔隙并未发生明显变形, 介质变形主要体现为孔隙周边基体的微塑性(剪切变 形)和开裂(横向拉应变), 以及孔隙周边开裂区域的相 互连通(文献[21]CT 扫描实验也发现了类似现象). 基体 大范围的破裂和相互连通发生在应力波峰越过孔隙介 质进入透射杆传播时, 残余作用压应力约为峰值应力 的 48\% 52\%. 基于孔隙介质三维模型的有限元模拟可 以直观定量地显示应力波传播过程中岩石介质内部孔 隙和基体的应力、应变状态及变形破坏机制, 而宏观波 动实验(包括 SHPB 实验)是难以刻画这种内部机制的.

\section{6 孔隙介质的能量耗散}

应力波穿越孔隙介质时，由于孔隙周围基体开 
裂和微塑性等内部机制, 孔隙介质会消耗入射波能 量. 能量耗散规律反映了应力波传播过程中孔隙介 质内部结构的不可逆变化趋势, 有助于解析孔隙介 质复杂的波传播性质和动力响应特征.

假设压杆中的应力波为一维弹性波，传播过程 无弥散无畸变, 杆端横截面在变形及运动中保持为 平面. 被测样品的应力及应变均匀(宏观尺度上). 设 $A_{0}, E_{0}, C_{0}$ 和 $\rho_{0}$ 分别为压杆的横截面积、弹性模量、纵 波波速和材料密度. 根据 SHPB 波动理论 ${ }^{[21,34 ~ 39]}$, 压 杆中入射波、反射波和透射波的能量可分别表示为

$$
\begin{aligned}
& W_{I}=\frac{A_{0}}{c_{0} \rho_{0}} \int_{0}^{t} \sigma_{I}^{2}\left(t^{\prime}\right) \mathrm{d} t^{\prime}, \\
& W_{R}=\frac{A_{0}}{c_{0} \rho_{0}} \int_{0}^{t} \sigma_{R}^{2}\left(t^{\prime}\right) \mathrm{d} t^{\prime}, \\
& W_{T}=\frac{A_{0}}{c_{0} \rho_{0}} \int_{0}^{t} \sigma_{T}^{2}\left(t^{\prime}\right) \mathrm{d} t^{\prime},
\end{aligned}
$$

式中: $W_{I}, W_{R}$ 和 $W_{T}$ 代表入射波、反射波和透射波的能 量. $\sigma_{I}(t), \sigma_{R}(t)$ 和 $\sigma_{T}(t)$ 为入射波、反射波和透射波的 应力时程, 由应变时程 $\varepsilon_{j}(t)$ 按照弹性波理论计算. 波 速 $c_{0}=\left(E_{0} / \rho_{0}\right)^{1 / 2}$. 代入数值模拟或者物理实验得到 的压杆长 $1 / 2$ 位置处的入射波、反射波和透射波应变 时程 $\mathcal{E}_{j}(t)$ 可以求出波动能量 $W_{I}, W_{R}$ 和 $W_{T}$. 假设应力 波传播为等温过程，与外部没有热交换. 忽略试件两 端部与压杆的摩擦作用, 根据热力学第一定律, 孔隙 介质应力波动过程中的能量满足以下关系

$$
\begin{aligned}
& W_{I}=W_{R}+W_{J}+W_{T}, \\
& W_{J}=W_{I}-W_{R}-W_{T},
\end{aligned}
$$

其中, $W_{J}$ 表示孔隙介质所耗散的能量, 为不可逆耗散能. 利用公式(4) (8), 代入应变时程 $\varepsilon_{j}(t)$ 进行积分运算可以 得出耗散能 $W_{J}$. 为了说明不可逆变形对介质耗能能 力的影响, 我们计算了不同孔隙率介质的耗散能 $W_{J}$ 占 全部输入能量 $W_{I}$ 的比值 $W_{J} / W_{I}$, 即能量耗散率. 图 20 给出了冲击速度 $16.9 \mathrm{~m} / \mathrm{s}$ (应变率 $\dot{\varepsilon} \leq 90 / \mathrm{s}$ ) 条件下介 质能量耗散率 $W_{J} / W_{I}$ 随孔隙率 $\rho_{v}$ 变化的趋势及相关拟 合函数. 作为对比, 图 20 中同时给出了根据物理模 型实验得到的应变时程计算的能量耗散率.

计算结果表明:

(i) 恒定冲击速度下孔隙介质的能量耗散率 $W_{J} / W_{I}$ 随孔隙率 $\rho_{v}$ 增大而线性增加. 表明岩石类介质 中孔隙数量越多, 介质不可逆变形越大. 数值模拟与 物理模型实验得到了相同的能量耗散率的演化趋势.

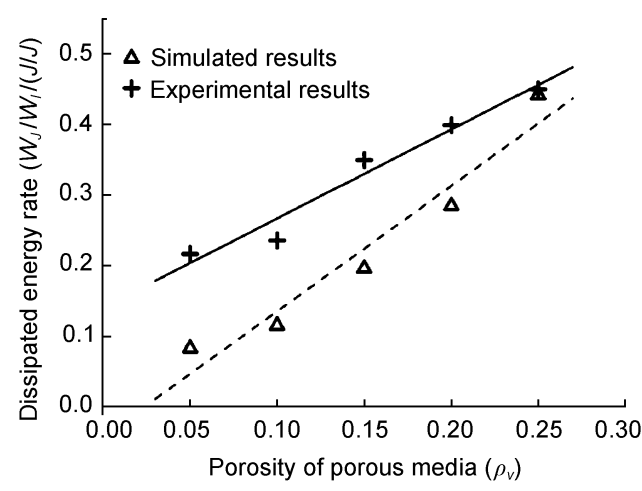

图 20 孔隙介质能量耗散率 $W_{J} / W_{I}$ 随孔隙率 $\rho_{v}$ 变化 的规律(冲击速度 $16.9 \mathrm{~m} / \mathrm{s}$ )

参照文献[21], 岩石类孔隙介质能量耗散率 $W_{J} / W_{I}$ 随 孔隙率 $\rho_{v}$ 线性变化的关系可表示为

$$
W_{J} / W_{I}=A+B \cdot \rho_{v},
$$

式中 $A, B$ 为与冲击速度或应变率有关的材料参数, 可以通过冲击实验得到.

(ii) 相同孔隙率条件下, 能量耗散率 $W_{J} / W_{I}$ 的计算 值低于相应的实验值，计算值与实验值之间存在一定 的偏差. 孔隙率越大，两者越接近，偏差越小。

\section{7 结语}

通过提取天然砂岩的孔隙结构参数、矿物组成和 物理力学性质, 制作了具有与天然砂岩相同的孔隙 结构特征和基体性质、但孔隙率不同的孔隙介质物理 模型. 利用物理模型的 CT 扫描图像和 Mimics ${ }^{\circledR}$ 构建 了具有不同孔隙率的孔隙结构三维有限元模型. 通 过设定应力波动理论假设的条件模拟了孔隙介质 SHPB 冲击破坏过程, 分析了波动应力作用下岩石类 孔隙介质的动力学响应、应力传递规律与变形破坏机 制. 研究获得以下发现和结论.

(i) 孔隙介质三维模型和应力波动有限元模型可 以直观定量地刻画应力波传播过程中岩石类介质内 部孔隙和基体的应力、应变状态及变形破坏机制. 模 拟得到的孔隙介质的应力波传播规律、变形和破坏模 式以及能量耗散性质与物理模型的实验结果相吻合. 但由于实际实验条件和数值模拟条件的差异, 模拟 的应力波形和能量耗散率与实验结果之间存在一定 偏差.

（ii）相同冲击速度条件下，随着介质孔隙率增大， 
反射波幅增加, 透射波幅减小. 高应力/高应变区和 破坏区集中分布在试件侧面以及两端面部分孔隙的 周边基体. 在入射波和反射波的叠加作用下, 孔隙周 围的基体单元产生较大的剪应力和拉应力, 剪应力 使基体单元产生微塑形(剪切变形), 拉应力使基体单 元产生开裂(横向拉变形). 孔隙周边基体单元的破坏 与相互贯通主要是由于基体单元的横向拉应力/拉应 变超过材料极限值而造成的. 孔隙介质物理模型的 SHPB 冲击实验与内部结构破坏形态的 CT 扫描实验 证实了上述模拟分析.

(iii) 一定压强和波速的应力波传播过程中(冲击速 度 $v_{0} \leq 16.9 \mathrm{~m} / \mathrm{s}$, 应变率 $\left.\dot{\varepsilon} \leq 90 / \mathrm{s}\right)$, 孔隙率低于 $15 \%$ 的 岩石介质的内部孔隙未发生明显变形, 介质变形主要 体现为孔隙周边基体的微塑性(剪切变形)和开裂(横 向拉应变), 以及孔隙周边开裂区域的相互连通. 基 体大范围的破裂和相互连通发生在应力波峰越过孔隙 介质进入透射杆转播时, 残余作用压应力约为峰值应 力的 $48 \%$ - 52\%.

(iv) 应力波传播过程中, 当压应力波逐步离开 孔隙试件进入透射杆传播后, 孔隙介质基体产生弹 性变形恢复, 对入射杆产生一个反向的压缩应力, 该
压缩波与入射杆和介质交界面处产生的反射拉伸波 相互叠加, 造成入射杆上记录的反射波波长小于入 射波波长. 孔隙率越小, 反射波波幅越小, 波长越短. 孔隙介质物理模型的 SHPB 实验也证实了这一点.

(v) 恒定冲击速度下孔隙介质的能量耗散率 $W_{J} / W_{I}$ 随孔隙率 $\rho_{v}$ 增大而线性增加. 数值模拟与物理 模型实验得到了相同的能量耗散率的演化趋势. 表 明岩石类孔隙介质中孔隙数量越多, 基体发生的微 塑性和开裂等不可逆变形越大. 岩石类孔隙介质能 量耗散率 $W_{J} / W_{I}$ 随孔隙率 $\rho_{v}$ 变化的关系可采用线性方 程表示.

需要指出的是, 作为初步尝试, 本文构建了岩石 类孔隙介质的三维有限元模型, 并利用该模型模拟 研究了应力波传播过程中岩石介质内部孔隙和基体 的应力、应变状态及变形破坏机制, 为探索和揭示岩 石类孔隙介质的复杂多变动力学行为的内在机理、应 力传递规律、变形破坏与致灾机理提供了参考. 但限 于篇幅, 本文未就冲击速度(或应变率)、孔隙参数、 固体相性质等因素对孔隙介质应力波动性质和内部 变形破坏机制的影响展开深入讨论，有关这方面的 分析作者将另文介绍.

\section{参考文献}

1 姜耀东, 赵毅金金, 刘文岗, 等. 煤岩冲击失稳的机理和实验研究. 北京: 科学出版社, 2009

2 Lasocki S, Orlecka-Sikora B. Seismic hazard assessment under complex source size distribution of mining-induced seismicity. Tectonophys, 2008, 456: $28-37$

3 Heuze F E, Morris J P. Insights into ground shock in jointed rocks and the response of structures there-in. Int J Rock Mech Min Sci, 2007, 44: $647-676$

4 Qin S Q, Jiao J J, Tang C A, et al. Instability leading to coal bumps and nonlinear evolutionary mechanisms for a coal-pillar-and-roof system. Int J Solids Struct, 2006, 43: 7407-7423

5 Hazzard J F, Young R P. Dynamic modelling of induced seismicity. Int J Rock Mech Min Sci, 2004, 41: 1365-1376

6 潘一山, 王来贵, 章梦涛, 等. 断层冲击地压发生的理论与试验研究. 岩石力学与工程学报, 1998, 17: 642一 649

7 Beamish B B, Crosd B P J. Instantaneous outbursts in underground coal mines: An overview and association with coal type. Int J Coal Geology, 1998, 35: $27-55$

8 Tang C A, Kaiser P K. Numerical simulation of cumulative damage and seismic energy release during brittle rock failure Part 1: Fundamentals. Int J Rock Mech Min Sci, 1998, 35: 123-134

9 Fujii Y, Ishijima Y, Deguchi G. Predication of coal face rock burst and micro-seismicity in deep longwall coal mine. Int J Rock Mech Min Sci, 1997, 34: 85-96

10 Boler F M, Billington S, Zipf R K. Seismological and energy balance constraints on the mechanism of a catastrophic bump in the Book Cliffs coal mining district, Utah, USA. Int J Rock Mech Min Sci Geomech Abstr, 1997, 34: 27—43

11 Xie H. Fractals in rock mechanics. A Balkema, Rotterdam, 1993

12 Lippmann H. Keynote lecture: Mechanical considerations of bumps in coal mines. In: Proceeding 2nd Int Symp. Rockbursts and Seismicity in Mines, A Balkema, 1990

13 Carcione J M, Helle H B, Santos J E, et al. A constitutive equation and generalized Gassmann modulus for multimineral porous media. Geophys, 
2005, 70: N17-N26

14 Sharma M D. Three-dimensional wave propagation in a general anisotropic poroelastic medium: Phase velocity, group velocity and polarization. Geophys J Int, 2004, 156: 329-344

15 Sharma M D. Surface waves in a general anisotropic poroelastic solid half-space. Geophys J Int, 2004, 159: 703-710

16 汪越胜, 于桂兰, 章梓茂, 等. 复杂界面(界面层)条件下的弹性波传播问题研究综述. 力学进展, 2000, 30: 378一 390

17 Crampin S. The fracture criticality of crustal rocks. Geophys J Int, 1994, 118: 428-438

18 Vardoulakis I, Muhlhaus H B. Local rock surface instabilities. Int J Rock Mech Min Sci Geomech Abstr, 1986, 23: 379-383

19 Kraut E A. Advances in the theory of anisotropic elastic wave propagation. Rev Geophys, 1963, 1: 401-448

20 Biot M A. The theory of propagation of elastic waves in a fluid- saturated porous solids I. Low-frequency range, II. High frequency range. J Acoust Soc Am, 1956, 28: 168一191

21 鞠杨, 杨永明, 毛彦喆, 等. 孔隙介质中应力波传播机制的实验研究. 中国科学 $\mathrm{E}$ 辑: 技术科学, 2009, 39: 904一 918

22 Lambert G, Gurevich B, Brajanovski M. Attenuation and dispersion of P-waves in porous rocks with planar fractures: Comparison of theory and numerical simulations. Geophys, 2006, 71: N41-N45

23 Mashinskii E I. Experimental study of the amplitude effect on wave velocity and attenuation in consolidated rocks under confining pressure. J Geophys Engng, 2005, 2: 199-212

24 Fratta D, Santamarina J C. Shear wave propagation in jointed rock: State of stress. Geotech, 2002, 52: 495-505

25 刘凯欣, 刘颖. 液饱和多孔介质三维应力波的传播. 力学学报, 2003, 35: 469-473

26 Arntsen B, Carcione J M. Numerical simulation of the Biot slow wave in water-saturated Nivelsteiner sandstone. Geophys, 2001, 66: 890-896

27 Kelner S, Bouchon M, Coutant O. Numerical simulation of the propagation of P waves in fractured media. Geophys J Int, 1999, 137: 197206

28 Cheng N. Nonlinear wave propagation in sandstone: A numerical study. Geophys, 1996, 61: 1935-1938

29 Carcione J M. Wave propagation in anisotropic, saturated porous media: Plane-wave theory and numerical simulation. J Acoust Soc Am, 1996, 99: 2655-2666

30 TenCate J A, Van Den Abeele K E A, Shankland T J, et al. Laboratory study of linear and nonlinear elastic pulse propagation in sandstone. J Acoust Soc Am, 1996, 100: 1383-1391

31 Nagy G, Murakami H, Hegemier G A, et al. Experimental and analytical study of the dynamic response of low-porosity brittle rocks. J Geophys Res, 1993, 98: 22081—22094

32 Krishnamoorthy K, Goldsmith W, Sackman J L. Measurements of wave processes in isotropic and transversely isotropic elastic rocks. Int J Rock Mech Min Sci Geomech Abstr, 1974, 11: 367-378

33 鞠杨, 杨永明, 宋振铎, 等. 岩石孔隙结构的统计模型. 中国科学 $\mathrm{E}$ 辑: 技术科学, 2008, 38: 1026-1041

34 Frew D J, Forrestal M J, Chen W. A split Hopkinson pressure bar technique to determine compressive stress-strain data for rock materials. Exper Mech, 2001, 41: 40-46

35 Dioh N N, Ivankovic A, Leevers P S, et al. Stress wave propagation effects in split Hopkinson pressure bar tests. Proc Royal Society London, Math Phys Sci-Ser A, 1995, 449: 187-204

36 Gorham D A, Pope P H, Field J E. An improved method for compressive stress-strain measurements at very high strain rates. Proc Royal Society London, Math Phys Sci-Ser A, 1992, 438: 153-170

37 Lindholm U S. Some experiments with split Hopkinson pressure bar. J Mech Phys Solids, 1964, 12: 317—335

38 Davies E D H, Hunter S C. Dynamic compression testing of solids by method of split Hopkinson pressure bar. J Mech Phys Solids, 1963, 11: 155-179

39 王会杰. 孔隙岩石动态力学性能与变形破坏机制的试验研究. 硕士学位论文. 北京: 中国矿业大学, 2009. 13-38

40 杨永明. 岩石孔隙结构模型与力学性能研究. 博士学位论文. 北京: 中国矿业大学, 2008. 59-84 